\title{
Use of Low- and High-Energy Collision- Induced Dissociation Tandem Mass Spectrometry in the Identification of an Unusual Amino Acid in a Semisynthetic Polypeptide
}

\author{
Ioannis A. Papayannopoulos \\ Biogen, Inc., Cambridge, Massachusetts, USA
}

\begin{abstract}
During production of the semisynthetic eicosapeptide Phe-Pro-Arg-Pro-Gly-Gly-Gly-GlyAsn-Gly-Asp-Phe-Glu-Glu-Ile-Pro-Glu-Glu-Tyr-Leu, two minor impurities were isolated in which Ile-15 had been replaced by other amino acids. The molecular weights of the two peptide minor products were $14 \mathrm{u}$ lower and $14 \mathrm{u}$ higher, respectively, than the major product with the foregoing amino acid sequence. It was determined that the first, lower molecular weight, impurity contained Val instead of lle at position 15 of the sequence, whereas the second, higher molecular weight, impurity contained an unusual amino acid at the same position. This amino acid, which was characterized from the low- and high-energy collision-induced dissociation product ion tandem mass spectra of the second peptide impurity, is an $\alpha$-amino acid with an asymmetric side chain branched at the $\beta$-carbon. (J Am Soc Mass Spectrom 1996, 7, 1034-1039)
\end{abstract}

$\mathrm{M}$ ass spectrometry and tandem mass spectrometry have been used extensively in the elucidation of modifications to the sequence of natural, recombinant, and synthetic proteins and peptides [1-3]. Such modifications sometimes involve the substitution of one amino acid by another, either because the protein or peptide being analyzed, rather than being pure, is a mixture of closely related multiple gene products [4] or because an amino acid other than 1 of the 20 common protein amino acids was incorporated into the sequence during biosynthesis (e.g., norleucine in the place of methionine [5]). Molecular weight information is often the first mass spectrometric indication that such a modification has occurred; tandem mass spectrometry data, and, specifically, collision-induced dissociation (CID) product ion mass spectra, can be used to pinpoint the location of the modification and obtain structural information for the unexpected amino acid.

The eicosapeptide Phe-Pro-Arg-Pro-Gly-Gly-GlyGly-Asn-Gly-Asp-Phe-Glu-Glu-Ile-Pro-Glu-Glu-TyrLeu was made semisynthetically by production of the octadecapeptide Arg-Pro-Gly-Gly-Gly-Gly-Asn-GlyAsp-Phe-Glu-Glu-Ile-Pro-Glu-Glu-Tyr-Leu in E. coli, isolation and purification, and concatenation at the $\mathrm{N}$-terminus with the synthetically made dipeptide Phe-Pro. During reversed-phase high-performance liquid chromatographic (HPLC) analysis of the product,

Address reprint requests to Dr. I. A. Papayannopoulos, Biogen Inc., 14 Cambridge Center, Cambridge, MA 02142. in addition to the main component, with a retention time corresponding to that of the target eicosapeptide, two minor components, each about $1 \%$ of the major component, also were detected. The HPLC peaks that correspond to the two minor impurities were collected and sequenced by automated Edman degradation. Each peak contained a peptide with the correct amino acid sequence except for position 15, where, in the first peptide impurity, the expected Ile residue had been replaced by Val, whereas in the second, the Ile residue had been replaced by another amino acid that did not correspond to any of the standard amino acids with which the sequencer had been calibrated.

Fast-atom bombardment (FAB) [6, 7] mass spectrometric analysis of the two impurities (Figure 1) revealed that the first contained a peptide with monoisotopic molecular weight 2165.1, which is in agreement with the calculated molecular weight of 2165.0 for the eicosapeptide Phe-Pro-Arg-Pro-Gly-Gly-Gly-Gly-AsnGly-Asp-Phe-Glu-Glu-Val-Pro-Glu-Glu-Tyr-Leu that had been identified by $\mathrm{N}$-terminal Edman sequencing. The molecular weight of the peptide that constituted the second impurity was 2193.1, 14 u higher than the molecular weight calculated for the eicosapeptide with the expected sequence and $28 \mathrm{u}$ higher than the molecular weight of the first peptide impurity. Taken together, the molecular weight and $\mathrm{N}$-terminal sequence data for the second peptide impurity suggest that the amino acid at position 15 has a residue mass of 127 (14 $\mathrm{u}$ higher than Ile), which does not correspond to any natural amino acid. 


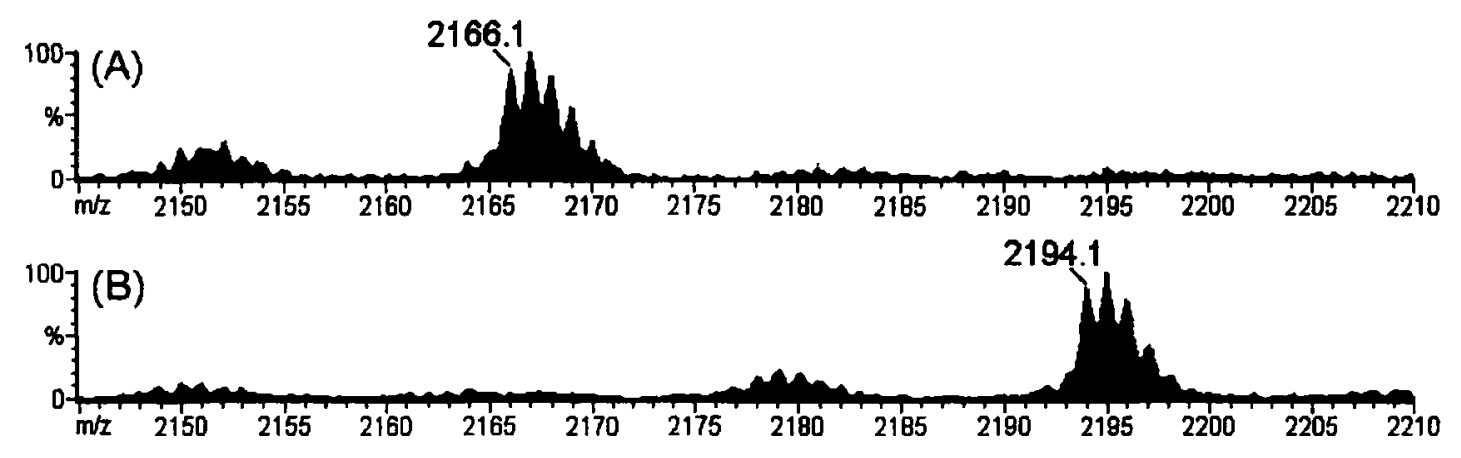

Figure 1. Partial FAB mass spectrum of the protonated peptide molecule $\left([\mathrm{M}+\mathrm{H}]^{+}\right)$regions of the two minor impurities generated during the production of the eicosapeptide Phe-Pro-Arg-Pro-GlyGly-Gly-Gly-Asn-Gly-Asp-Phe-Glu-Glu-De-Pro-Glu-Glu-Tyr-Leu (calculated ${ }^{12} \mathrm{C}$ monoisotopic molecular weight 2179.98). The two samples were separated by HPLC. One peptide impurity (A) has a molecular weight $14 \mathrm{u}$ lower than expected, whereas the other (B) has a molecular weight $14 \mathrm{u}$ higher than expected. The major component (spectrum not shown) exhibits a $[\mathrm{M}+\mathrm{H}]^{+}$at $m / z$ 2180.1 .

To confirm the presence of Val-15 in the first peptide impurity and characterize the amino acid present at the same position in the sequence of the second, collision-induced dissociation (CID) product ion tandem mass spectrometry [8-10] was employed.

\section{Experimental}

$\mathrm{FAB}^{*}$ and low-energy CID product ion tandem mass spectra were acquired with a VG Quattro II triple quadrupole mass spectrometer (Fisons Instruments, Altrincham, England). Secondary ions were generated by bombardment of the sample (dissolved in glycerol) with cesium ions. The sample concentration in the glycerol matrix was approximately $0.5 \mathrm{nmol} / \mu \mathrm{L}$. The first quadrupole was operated at unit mass resolution, which allowed selection of only the ${ }^{12} \mathrm{C}$ isotope peak from the singly protonated molecule isotope cluster and transmission into the collision region. The collision gas (argon) was introduced into the collision region at a pressure sufficient to reduce the precursor ion signal by approximately three quarters. The collision energy was $40 \mathrm{eV}$. The instrument was calibrated for mass spectrometry and tandem mass spectrometry operation with a mixture of CsI and NaI. All data were acquired in profile mode by using the VG MassLynx data system (Fisons Instruments), where each product ion mass spectrum comprised the sum of 5-6 scans.

High-energy CID product ion tandem mass spectra were obtained with a JEOL HX110/HX110 magnetic deflection tandem mass spectrometer (JEOL, Akishima, Japan). Secondary ions were generated from the sample by cesium ion bombardment and were accelerated to $10 \mathrm{keV}$. The collision cell potential was 3 $\mathrm{kV}$, which resulted in a collision energy of $7 \mathrm{keV}$. The collision gas (helium) was introduced into the collision cell at a pressure such that the precursor ion signal

\footnotetext{
*This term is used generically to describe the production of secondary ions from a sample dissolved in a liquid matrix (e.g., glycerol), by bombardment either with kiloelectronvolt neutral atoms (FAB) or kiloelectronvolt ions (liquid secondary ionization mass spectrometry).
}

was reduced by approximately two thirds. The first mass spectrometer was operated at a resolution sufficient so that only the ${ }^{12} \mathrm{C}$ isotope from the singly protonated molecule isotope cluster could be selected and transmitted into the collision cell. The instrument was calibrated for mass spectrometry operation with CsI and for tandem mass spectrometry operation with a mixture of alkali metal salts (for more details on the operation of the four-sector tandem mass spectrometer, see ref 11). Data were acquired in profile mode by using the JEOL DA7000 data system; two scans were summed for each product ion mass spectrum.

\section{Results and Discussion}

The low-energy tandem CID mass spectrum of the major product (the eicosapeptide Phe-Pro-Arg-ProGly-Gly-Gly-Gly-Asn-Gly-Asp-Phe-Glu-Glu-Ile-ProGlu-Glu-Tyr-Leu) is shown in Figure 2A; assignments of the major fragment ions for this spectrum are listed in Table 1. The tandem CID mass spectra of the two impurities, one $14 \mathrm{u}$ lower and the other $14 \mathrm{u}$ higher in mass than the major product, are shown in Figure 2B and $C$, respectively. All three spectra exhibit essentially the same fragment ions up to $m / z 1434$. Beyond this ion the mass-to-charge ratio values of the fragment ions in the tandem mass spectra of the two impurities are consistently $14 \mathrm{u}$ lower for the first and $14 \mathrm{u}$ higher for the second, compared with the mass-to-charge ratio values for the corresponding fragment ions of the major component. As is apparent from the fragment ion assignments in Table 1, upon CID these peptides fragment so that only ions that contain the peptide $\mathrm{N}$-terminus are produced, presumably because of the arginine residue at position 3 and the peptide $\mathrm{N}$-terminal amino group; these are the only basic sites in the sequence and, therefore, serve to localize the positive charge and to direct fragmentation [10, 12]. The last fragment ion that appears at the same mass-to-charge ratio (1434) in all three spectra is $c_{14}$ (see ref 5 for a discussion of peptide fragmentation and the structures 
Phe-Pro-Arg-Pro-Gly-Gly-Gly-Gly-Asn-Gly-Asp-Phe-Glu-Glu-Ile-Pro-Glu-Glu-Tyr-Leu
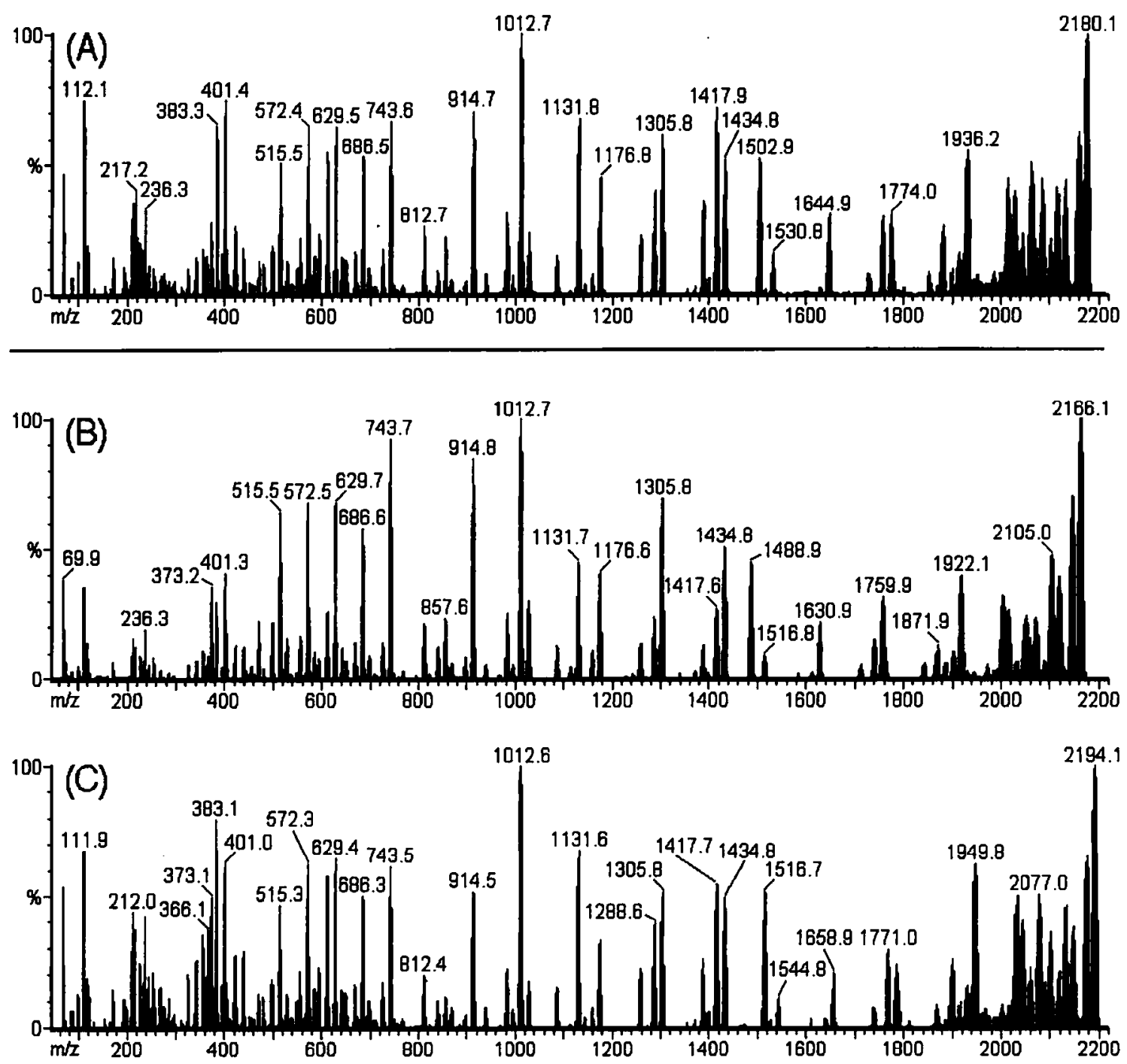

Figure 2. Tandem CID product ion mass spectra of the major component $(A)$ and the two minor peptide impurities (B) and (C). The $\left[\mathrm{M}+\mathrm{H}^{+}\right.$regions from the FAB mass spectra of the latter two are shown in Figure 1. The data are summarized in Table 1. All three spectra were acquired with a triple quadrupole instrument.

of fragment ions), and this, as well as the other $\mathrm{N}$ terminal fragment ions below it, shows that the first 14 amino acids are the same in all three peptides. For the expected 15th amino acid (Пle), the calculated mass-tocharge ratio values for ions $a_{15}$ and $b_{15}$ are 1502.70 and 1530.70 , respectively. The tandem mass spectrum of the major peptide product does, indeed, contain fragment ions at these mass-to-charge ratio values (1502.9 and 1530.8). However, in the spectrum of the first minor peptide impurity, the corresponding ions are at mass-to-charge ratio values $14 \mathrm{u}$ lower (1488.9 and 1516.8), whereas in the spectrum of the second minor impurity, these ions are at mass-to-charge ratio values $14 \mathrm{u}$ higher (1516.7 and 1544.8). This observation is consistent with the differences in molecular weights between the major product and the two impurities, and shows that the first peptide impurity contains, at position 15 of its sequence, an amino acid $14 \mathrm{u}$ lower in mass than Ile (residue mass 99 for this amino acid versus 113 for lle), whereas the second peptide impurity contains an amino acid $14 \mathrm{u}$ higher in mass than Ile at position 15 (residue mass 127 for this amino acid versus residue mass 113 for Ile). The remainder of the fragment ions in the tandem mass spectra of both minor peptide impurities are, respectively, consistently $14 \mathrm{u}$ lower and $14 \mathrm{u}$ higher in mass than the calculated mass-to-charge ratio values and correspond to the expected amino acids at positions 16-20 (see Table 1).

The data for the first peptide impurity are also in agreement with the $\mathrm{N}$-terminal sequence data previously mentioned, given that Val, identified at cycle 15 by the Edman method, is indeed $14 \mathrm{u}$ lower in mass than Ile. For the second impurity the situation is more complicated, because no natural amino acid is $14 \mathrm{u}$ higher in mass than Ile. The presence of an unusual or modified amino acid at position 15 in the sequence of 
Table 1. Calculated low-energy fragment ion mass-to-charge ratio values for the eicosapeptide Phe-Pro-Arg-Pro-Gly-Gly-Gly-GlyAsn-Gly-Asp-Phe-Glu-Glu-De-Pro-Glu-Glu-Tyr-Leu and the corresponding measured values for the major component and the two peptide impurities

\begin{tabular}{|c|c|c|c|c|c|}
\hline $\begin{array}{l}\text { Amino } \\
\text { acid }\end{array}$ & $\begin{array}{l}\text { Fragment } \\
\text { ion(s) }\end{array}$ & $\begin{array}{l}\text { Calculated } \\
\mathrm{m} / \mathrm{z}\end{array}$ & Major product & $\begin{array}{l}\text { First minor } \\
\text { inpurity }\end{array}$ & $\begin{array}{l}\text { Second minor } \\
\text { impurity }\end{array}$ \\
\hline \multicolumn{6}{|l|}{ Phe } \\
\hline Pro & $a_{2}$ & 217.13 & 217.2 & & \\
\hline Arg & $b_{3}$ & 401.23 & 401.4 & 401.3 & 401.0 \\
\hline \multicolumn{6}{|l|}{ Pro } \\
\hline Gly & $c_{5}$ & 572.33 & 572.4 & 572.5 & 572.3 \\
\hline Gly & $c_{6}$ & 629.35 & 629.5 & 629.7 & 629.4 \\
\hline Gly & $c_{7}$ & 686.37 & 686.5 & 686.6 & 686.3 \\
\hline Gly & $c_{8}$ & 743.40 & 743.6 & 743.7 & 743.5 \\
\hline \multirow[t]{2}{*}{ Asn } & $a_{9}$ & 812.42 & 812.7 & 812.6 & 812.4 \\
\hline & $c_{9}$ & 857.44 & 857.7 & 857.6 & \\
\hline Gly & $c_{10}$ & 914.46 & 914.7 & 914.8 & 914.5 \\
\hline Asp & $b_{11}$ & 1012.46 & 1012.7 & 1012.7 & 1012.6 \\
\hline \multirow[t]{2}{*}{ Phe } & $a_{12}$ & 1131.53 & 1131.8 & 1131.7 & 1131.6 \\
\hline & $c_{12}$ & 1176.56 & 1176.8 & 1176.6 & 1176.6 \\
\hline Glu & $c_{13}$ & 1305.60 & 1305.8 & 1305.8 & 1305.8 \\
\hline \multirow[t]{2}{*}{ Glu } & $b_{14}$ & 1417.61 & 1417.9 & 1417.6 & 1417.7 \\
\hline & $c_{14}$ & 1434.64 & 1434.8 & 1434.8 & 1434.8 \\
\hline \multirow[t]{2}{*}{ Ile } & $a_{15}$ & 1502.70 & 1502.9 & $1488.9^{b}$ & $1516.7^{c}$ \\
\hline & $b_{15}$ & 1530.70 & 1530.8 & $1516.8^{b}$ & $1455.8^{c}$ \\
\hline Pro & $c_{16}$ & 1644.78 & 1644.9 & $1630.9^{b}$ & $1658.9^{c}$ \\
\hline \multirow[t]{2}{*}{ Glu } & $b_{17}$ & 1756.79 & 1756.9 & $1743.0^{b}$ & $1771.0^{c}$ \\
\hline & $c_{17}$ & 1773.82 & 1774.0 & $1759.9^{b}$ & $1787.9^{c}$ \\
\hline Glu & $b_{18}$ & 1885.84 & 1886.1 & $1871.9^{\circ}$ & $1899.9^{c}$ \\
\hline \multicolumn{6}{|l|}{ Tyr } \\
\hline \multicolumn{6}{|l|}{ Leu } \\
\hline$[\mathrm{M}+\mathrm{H}]^{+} m / z$ & & 2179.99 & 2180.1 & 2166.1 & 2194.1 \\
\hline
\end{tabular}

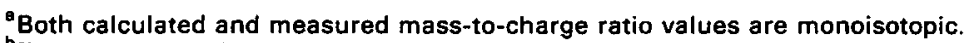

b These mass-to-charge ratio values are $14 \mathrm{u}$ lower than the calculated values for the correct peptide sequence.

"These mass-to-charge ratio values are $14 \mathrm{u}$ higher than the calculated values for the correct peptide sequence.

the second minor peptide impurity, however, is also consistent with the sequence data, because no known amino acid could be identified at cycle 15 .

The possibility that the second peptide impurity contains, at position 15 , an Ile residue with a $\mathrm{N}$-methyl $\alpha$-amino group (a modification that would add $14 \mathrm{u}$ to the peptide mass) can be dismissed easily because of the presence of the $c_{14}$ ion at $m / z$ 1434.8. Methylation of the Mle-15 $\alpha$-amino group (Figure $3 \mathrm{~A}$ ) would have resulted in a $c_{14}$ ion at $m / z 1448.65$, but no such ion can be found in the tandem mass spectrum of this peptide. It appears, therefore, that the most likely amino acid at position 15 is an $\alpha$-amino acid with a side chain that contains one methyl group more than Ile, that is, a 2-aminoheptanoic acid (Figure 3B).

To determine mass spectrometrically which one of several possible isomers the amino acid in question is, data on the structure of its side chain are necessary. Because amino acid side chain fragmentation processes occur in high-energy CID of peptides $[9,10,12,13]$, no

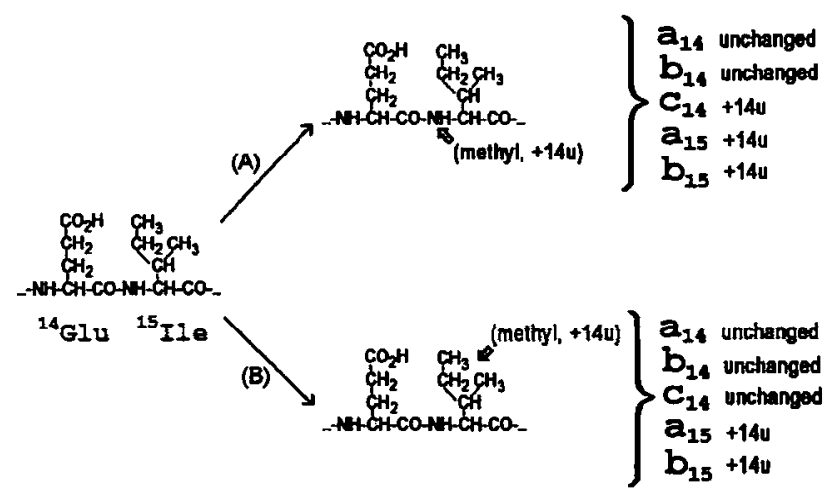

Figure 3. The two possible sites of attachment of a methyl group to the Ile residue at position 15 of the eicosapeptide Phe-Pro-Arg-Pro-Gly-Gly-Gly-Gly-Asn-Gly-Asp-Phe-Glu-Glu-IlePro-Glu-Glu-Tyr-Leu, which would result in a peptide with the same molecular weight as the second peptide impurity. The reasons why the first possibility (A), a posttranslational modification of Me-15, can be dismissed, whereas the second possibility (B), incorporation of an unusual amino acid at position 15 during biosynthesis, is supported by the data are discussed in the text. 


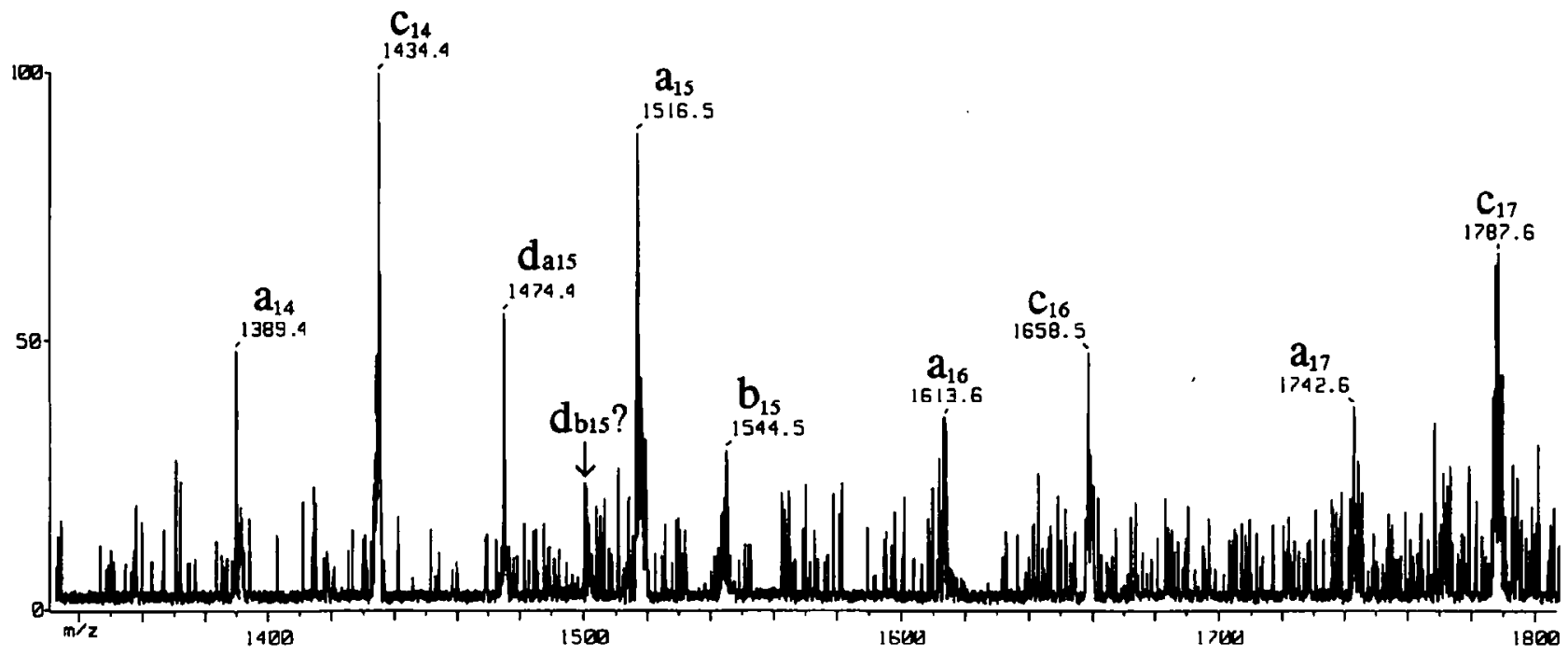

Figure 4. Partial high-energy CID product ion tandem spectrum of the higher molecular weight peptide impurity obtained with a four-sector magnetic deflection instrument. In addition to ions also present in the low-energy product ion spectrum (Figure 2C), ions that result from fragmentation of the asymmetrical side chain of the unusual amino acid at position 15 appear at $42 \mathrm{u}$ and $14 \mathrm{u}$ below the mass-to-charge ratio value of the corresponding $a$ ion $\left(d_{a 15}\right.$ and $d_{b 15}$, respectively).

such data were available in the low-energy CID product ion mass spectra shown in Figure 2 and discussed heretofore. Thus, the high-energy CID product ion mass spectrum of the second peptide impurity (protonated molecule $m / z$ 2194.1) was acquired. The region of this tandem mass spectrum that contains the ions necessary to address the issue of the structure of the side chain of the unusual amino acid at position 15 is shown in Figure 4. As with the low-energy tandem mass spectrometry data discussed in the foregoing text, the $c_{14}$ fragment ion at $m / z 1434.4$ (along with the $a_{14}$ ion at $m / z$ 1389.4) indicates that the first 14 amino acids of the sequence are as expected and that the $\alpha$-amino group of the amino acid at position 15 is not modified. The pair of ions at $\mathrm{m} / z 1516.5$ and 1544.5 can be assigned as $a_{15}$ and $b_{15}$; the former is 127 $\mathbf{u}$ (the residue mass of the unusual amino acid at position 15) higher in mass than the $a_{14}$ ion. The ion at $m / z 1474.4$ is $42 \mathrm{u}$ lower in mass than the $\mathrm{a}_{15}$ ion and it could be the $d_{15}$ ion that results from loss of a propyl (or isopropyl) group from the $a_{15}$ ion with transfer of a hydrogen atom (such high-energy fragmentations have been shown to occur for amino acids with nonaromatic substituents at the $\beta$-carbon $[9,12])$. The only structure consistent with the data would be an amino acid with two substituents at the $\beta$-carbon, one methyl group, and one propyl or isopropyl group, which yields two d-type fragment ions at $14 \mathrm{u}$ and $42 \mathrm{u}$ below the mass-to-charge ratio of $a_{15}$, by analogy with Ile, which has one methyl and one ethyl substituents at the $\beta$ carbon and yields two d-type fragment ions at $14 \mathrm{u}$ and $28 \mathrm{u}$ below the mass-to-charge ratio of the corresponding a ion [12]. The other possibility - an amino acid with a symmetrical side chain that has two ethyl substituents at the $\beta$-carbon-would yield one d ion,
$28 \mathrm{u}$ below the mass-to-charge ratio of $\mathrm{a}_{15}$, by analogy with Val, which has two methyl substituents at the $\beta$-carbon and yields one d-type fragment ion at $14 \mathrm{u}$ below the mass-to-charge ratio of the corresponding a ion; such an ion is not present in the spectrum. Although the signal-to-noise ratio in the tandem mass spectrum renders the unambiguous identification of the $d_{b 15}$ fragment ion (loss of the methyl group) at $\mathrm{m} / z 1502$ somewhat problematic, the ion at $\mathrm{m} / \mathrm{z} 1474.4$ (Figure 4) can be assigned readily as the $\mathrm{d}_{\mathrm{a} 15}$ fragment ion (loss of the propyl or isopropyl group). In fact, it is not unexpected that the loss of the propyl (or isopropyl) radical to generate the $d_{a 15}$ fragment ion would be more favorable than the loss of the methyl radical to generate the $d_{b 15}$ fragment ion, as is the case with Ile [12].

The high-energy CID product ion tandem mass spectral data, therefore, allow the identification of the unusual amino acid (2-aminoheptanoic acid) present at position 15 of the second minor peptide impurity as either 2-amino 3-methylhexanoic acid, or 2-amino 3,4dimethyl pentanoic acid (i.e., a methyl group and either a propyl or an isopropyl group attached to the $\beta$-carbon $\left.{ }^{\dagger}\right)$. These data are, of course, entirely consistent with the low-energy tandem mass spectrometry data, and are not in variance with the $\mathrm{N}$-terminal sequence data. Given the substitution of Val instead of Ile-15 in the other peptide impurity, it appears that

\footnotetext{
${ }^{\dagger}$ It should be pointed out that the molecular weight as well as the tandem mass spectrometry data are also consistent with oxidation of the Ile-15 side chain (specifically of the ethyl group attached to the $\beta$-carbon) to generate an aldehyde or ketone. However, the more complicated biochemical pathways required to effect this transformation and the presence of the other (Val-15-containing) minor impurity render this possibility less likely.
} 
under the specific conditions of cell growth, these two amino acids, which both have side chains branched at the $\beta$-carbon, were misidentified as Ile, bound by the isoleucyl-transfer RNA, and transported to the ribosomes where they were incorporated into the peptide chain during translation.

\section{Conclusions}

The use of product ion tandem mass spectrometry in the identification of amino acid posttranslational and other modifications in naturally occurring and synthetic peptides and proteins has been demonstrated repeatedly. Here it has been shown that although lowenergy CID tandem mass spectrometry data are sufficient to establish convincingly that an unusual amino acid was incorporated in the sequence of a peptide during biosynthesis, amino acid side chain fragmentation data, obtained from the high-energy tandem mass spectrum, are very useful to provide additional structural information necessary to distinguish between possible isomeric structures.

\section{Acknowledgments}

The high-energy CID product ion tandem mass spectrum was acquired with the four-sector tandem mass spectrometer installed at the NIH Mass Spectrometry Facility at MIT (Grant
RR00317 to K. Biemann). The N-terminal Edman of the two peptide impurities was determined by C. N. Young and E. P. Chow (Biogen, Inc.).

\section{References}

1. Biemann, K.; Scoble, H. A. Science 1987, 237, 992-998.

2. Carr, S. A.; Hemling, M. E.; Bean, M. F.; Roberts, G. D. Anal. Chem. 1991, 63, 2802-2824.

3. Hackett, M.; Guo, L.; Shabanowitz, J.; Hunt, D. F.; Hewlett, E. L. Science 1994, 266, 433-435.

4. Biemann, K.; Papayannopoulos, I. A. Acc. Chem. Res. 1994, $27,370-378$.

5. Trupin, J.; Dickerman, H.; Nirenberg, M.; Weissback, $\mathrm{H}$. Biochem. Biophys. Res. Commun. 1966, 24, 50-55.

6. Barber, M.; Bordoli, R. A.; Sedgewick, R. D.; Tyler, A. N. I. Chem. Soc., Chem. Commun. 1981, 325-327.

7. Dell, A.; Morris, H. R. Biochem. Biophys. Res. Commun. 1982, 106, 1456-1462.

8. Hunt, D. F.; Yates, J. R., III; Shabanowitz, J.; Winston, S.; Hauer, C. R. Proc. Natl. Acad. Sci. USA 1986, 83, 6233-6237.

9. Biemann, K. Biomed. Environ. Mass Spectrom. 1988, 16, 99-111.

10. Papayannopoulos, I. A. Mass Spectrom. Rev. 1995, 14, 49-73.

11. Wada, Y.; Matsuo, T.; Papayannopoulos, I. A.; Costello, C. E.; Biemann, K. Int. J. Mass Spectrom. Ion Processes 1992, 122, 219-229.

12. Johnson, R. S.; Martin, S. A.; Biemann, K. Int. J. Mass Spectrom. Ion Processes 1988, 86, 137-154.

13. Johnson, R. S.; Martin, S. A.; Biemann, K.; Stults, J. T.; Watson, J. T. Anal. Chem. 1987, 59, 2621-2625. 